\section{Bronchialkarzinom im Stadium I: Operation oder Radiotherapie?}

Spencer KL et al. Surgery or radiotherapy for stage I lung cancer? An intention to treat analysis. Europ Resp J 2019; PMID: 30635294. doi:10.1183/13993003.01568-2018

Beim Bronchialkarzinom im Stadium I stellt die chirurgische Resektion bei operablen Patienten die übliche Therapie dar. Ist eine Operation nicht möglich oder das perioperative Risiko zu hoch, bietet sich die stereotaktische fraktionierte Radiotherapie als Therapie an. Beide Verfahren zu vergleichen ist unter anderem wegen der gesundheitlich meist unterschiedlich fitten Probanden schwierig. Bisher ergaben sich widersprüchliche Ergebnisse.

Historische Serien einer konventionellen fraktionierten Bestrahlung beim Bronchialkarzinom im Stadium I bei nichtoperablen Patienten ergaben eine Unterlegenheit dieser Therapie gegenüber der Operation. Die stereotaktische ablative Radiotherapie (SABR) jedoch erlaubt deutlich höhere Intensitäten. Verschiedene Studien zeigten gleich gute Erfolge zwischen der chirurgischen Resektion und der SABR, eine kürzliche Untersuchung jedoch sprach der Operation die Überlegenheit zu. Schwierig ist der Vergleich beider Verfahren, weil die nichtoperablen Patienten eher jünger und gesünder sind als die anderen und sich bei der Bestrahlung das tatsächliche Tumorstadium nicht so eindeutig klären lässt wie durch eine Operation.
Um zu dieser Frage weitere Daten zu liefern, analysierten die Autoren dieser retrospektiven Studie die Daten aller Patienten des Universitätsklinikums Leeds, Großbritannien, die von 2008 bis 2013 wegen eines Bronchialkarzinom im Stadium I therapiert worden waren. In die Intention-to-treat-Analyse wurden 468 Patienten eingeschlossen, darunter 315 nach erfolgter chirurgischer Resektion und 99 nach SABR (sowie 53 nach konventioneller Strahlentherapie). Bei den operierten Patienten erfolgte in der Mehrheit eine Lobektomie oder Pneumonektomie. Die chirurgisch therapierten Patienten waren jünger und befanden sich in einem besseren Allgemeinzustand als die anderen. Die Autoren errechneten das Gesamtüberleben, das tumorspezifische Überleben und einen kombinierten Endpunkt aus tumor- und therapieassoziiertem Überleben.

Das 2-Jahres-Gesamtüberleben betrug

- 79,8\% für die Patienten nach Operation,

- 58,6\% nach SABR,

- 54,7\% nach konventioneller Radiotherapie.

Im Vergleich zur chirurgischen Resektion erwiesen sich dadurch beide radiologischen Verfahren in Bezug auf das Gesamtüberleben als signifikant weniger effektiv: Der Vergleich mit SABR ergab in der multivariaten Analyse eine Hazard Ratio von 1,84 (95\% Konfidenzintervall 1,317-2,570) und mit der konventionellen Bestrahlung eine Hazard Ratio von 2,278 (95\%-Konfidenzintervall 1,5393,372) (je $p<0,001$ ). In der univariaten Analyse waren folgende Faktoren mit einem kürzeren Gesamtüberleben assoziiert: höheres Alter, männliches Geschlecht, schlechter Allgemeinstatus, höheres Tumorstadium oder unbekannte Histologie. Alter und Geschlecht erwiesen auch in der multivariaten Analyse als Risikofaktoren.

Es ergaben sich jedoch keine Unterschiede zwischen Operation und SABR für das tumorspezifische Überleben (HR 1,469, $95 \%$ Konfidenzintervall 0,8-2,688) oder den kombinierten Endpunkt (HR 1,27, 95\%-Konfidenzintervall $0,744-2,17$ ). Bei den tumor- oder therapiebedingten Todesfällen zeigten sich keine Unterschiede zwischen den Behandlungsgruppen; Todesfälle, die unabhängig vom Tumor auftraten, kamen jedoch in multivariaten Analysen deutlich häufiger in der Gruppe nach Radiotherapie vor.

FAZIT

In dieser ersten Intention-to-treatAnalyse bei wahrscheinlichem Stadium-I-Bronchialkarzinom unterlag die Bestrahlung in Bezug auf das Gesamtüberleben der Operation. Allerdings zeigte die SABR beim tumorspezifischen Überleben gleich gute Ergebnisse wie die chirurgische Resektion. Randomisierte, kontrollierte Studien zu dieser Frage scheiterten an der Rekrutierung; nötig seien aber qualitativ hochwertige prospektive Daten, die Überleben und Lebensqualität der Patienten berücksichtigen, so die Autoren.

Dr. med. Susanne Meinrenken, Bremen 